Pacific

Journal of

Mathematics

QUANTITATIVE DEFORMATION THEOREMS AND CRITICAL POINT THEORY

JEAN-NoËL CORvelleC

Volume $187 \quad$ No. 2

February 1999 


\title{
QUANTITATIVE DEFORMATION THEOREMS AND CRITICAL POINT THEORY
}

\author{
JEAN-NoËL CORVElleC
}

\begin{abstract}
In the framework of critical point theory for continuous functionals defined on metric spaces, we show how quantitative deformation properties can be used to obtain saddle-point type results, even in the case when the usual geometric assumptions are not satisfied. We thus unify and extend to a nonsmooth setting some recent results of Schechter.
\end{abstract}

\section{Introduction.}

It is well known that deformation theorems are the basic tools in critical point theory. They can be derived under a condition of Palais-Smale type $\left((P S)\right.$, for short). In the classical setting of a $C^{1}$ functional $f$ defined on a Banach space $X$ (or a $C^{2}$ Finsler manifold), we refer to [15]; for a continuous functional $f$ defined on a complete metric space $X$, we refer to [8], the results of which include the case of a $C^{1}$ functional defined on a $C^{1}$ Finsler manifold. On the other hand, some authors gave, in the smooth case, so-called quantitative deformation theorems which are based on a weaker condition than $(P S)$, see, e.g., $[\mathbf{2}, \mathbf{2 1}]$, and also [16] for a detailed account of the theory.

Now, it is also well known how the min-max principle yields the existence of a critical value of the functional $f$, by combining a deformation theorem under the $(P S)$ condition, and some geometrical assumptions. Generally speaking, this is the case when a subset $B$ of $X$ links another subset $A$ of $X$, and the condition

$$
-\infty<b_{0}:=\sup _{B} f \leq \inf _{A} f=: a<+\infty
$$

is fulfilled, providing a critical level $b \geq a$ for $f$. On the other hand, in this geometrical situation, quantitative deformation theorems yield the existence of a Palais-Smale sequence for $f$ at the level $b$ - so that both approaches essentially provide the same information.

It turns out that the quantitative results allow to draw some conclusion, in the presence of linking, even in the case when (1.1) is not satisfied. Roughly speaking, and using the terminology introduced in [9], one can estimate the weak slope of $f$ at some point $u$ with $f(u) \in[a, b]$ in terms of the difference $b_{0}-a$ and the distance from $A$ and $B$; under appropriate assumptions, it 
is then possible to find a critical level for $f$ in the interval $[a, b]$. This idea is developed in the recent papers of Schechter $[\mathbf{1 7}, \mathbf{1 8}]$, dealing with $C^{1}$ functionals on Banach spaces (see also [20]).

In this paper, we extend this approach to saddle-point type results, in the context of the critical point theory of $[\mathbf{9}, \mathbf{8}]$. For this we use quantitative versions of the deformation theorems of [8], the former being straightforward consequences of the constructions performed in the latter. As for the notion of linking, we consider a variant of the one in [19], which proves convenient for our purposes, while allowing to recover all standard situations.

It is worth emphasizing that the deformations involved in our constructions are not homeomorphisms at each fixed time; indeed, when working in general metric spaces, we cannot expect this property to hold. Even in a Banach space setting, we do not know how to recover this property when dealing with functions which are only continuous. But, as it turns out, this is not needed to obtain essentially the desired generalizations of the results of $[\mathbf{1 7}, \mathbf{1 8}]$. On the other hand, working in metric spaces offers considerable freedom, as, for example, variants or extensions of the basic results are readily obtained by a simple change of metric. This is the case for the mentioned extensions of the results of [18]. Moreover, avoiding ad hoc constructions of deformations, all our results are deduced from the basic deformation theorems.

The results of this paper are developed in view of applications to nonsmooth variational problems, such as quasilinear elliptic problems of the type studied in $[\mathbf{3}, \mathbf{4}]$ (see also the references in $[4]$ ).

We mention that other deformation results for continuous functionals on metric spaces can be found in [6] (with an application in [7]), and in [12, 13] where a similar approach to nonsmooth critical point theory has been developed independently.

In Section 2, we recall the notion of weak slope and state our quantitative deformation theorems. The notion of linking is introduced in Section 3, and the main results on estimating the weak slope in the presence of linking are established. Section 4 deals with a general change-of-metric procedure, yielding extensions of the results of Section 3. Finally, some corollaries in Banach spaces are given in Section 5.

\section{Quantitative deformation theorems.}

In this section, $X$ is a metric space endowed with the metric $d$, and $f$ : $X \rightarrow \mathbb{R}$ is a continuous function. In the sequel, we denote respectively by $B(u ; \delta), \bar{B}(u ; \delta)$, and $\partial B(u ; \delta)$ the open, closed ball and sphere of radius $\delta>0$ centered at $u \in X$.

We start with recalling the notion of weak slope from [9], as well as the associated notions of Palais-Smale sequence and Palais-Smale condition. 
Definition 2.1 (See [9, Definition (2.1)]). For $u \in X$ we denote by $|d f|(u)$ the supremum of the $\sigma$ 's in $[0,+\infty[$ such that there exist $\delta>0$ and $\mathcal{H}$ : $B(u ; \delta) \times[0, \delta] \rightarrow X$ continuous with

$$
\begin{gathered}
d(\mathcal{H}(v, t), v) \leq t, \\
f(\mathcal{H}(v, t)) \leq f(v)-\sigma t .
\end{gathered}
$$

This notion was also introduced independently in [14], after a similar notion was defined in [12].

We say that $u \in X$ is a critical point of $f$ if $|d f|(u)=0$. For $c \in \mathbb{R}$, we let

$$
K_{c}=\{u \in X:|d f|(u)=0, f(u)=c\}
$$

denote the set of critical points of $f$ at level $c$.

We recall that if $X$ is a $C^{1}$ Finsler manifold and $f$ is of class $C^{1}$, then $|d f|(u)=\left\|f^{\prime}(u)\right\|$ for every $u \in X$. See [4] and the references therein for further comparisons between the weak slope and various analytical notions involving functionals which are not, in general, (Gâteaux) differentiable.

Definition 2.2. Let $f: X \rightarrow \mathbb{R}$ be a continuous function and let $c \in \mathbb{R}$. We say that a sequence $\left(u_{h}\right) \subset X$ is a Palais-Smale sequence for $f$ at level $c$ if $|d f|\left(u_{h}\right) \rightarrow 0$ and $f\left(u_{h}\right) \rightarrow c$. We say that $f$ satisfies the Palais-Smale condition at level $c\left((P S)_{c}\right.$ for short $)$, if every Palais-Smale sequence $\left(u_{h}\right)$ for $f$ at level $c$ contains a subsequence $\left(u_{h_{k}}\right)$ converging in $X$.

Since, clearly, $u \mapsto|d f|(u)$ is lower semicontinuous, a limit point of a Palais-Smale sequence for $f$ at level $c$ is a critical point of $f$ at level $c$. Condition $(P S)_{c}$ is used in critical point theory to obtain deformations properties of the sublevel sets of the function $f$, see [8, Theorems $(2.14),(2.15)]$. In fact, condition $(P S)_{c}$ readily implies the following, which is a condition of quantitative type used in practice to establish these deformation properties: Given $\gamma>0$ there exist $\delta>0$ and $\sigma>0$ such that

$$
c-\delta \leq f(u) \leq c+\delta, \quad d\left(u, K_{c}\right) \geq \gamma \quad \Longrightarrow \quad|d f|(u)>\sigma .
$$

Indeed, a close inspection of the proof of [8, Theorems (2.14), (2.15)] shows that the following two results hold.

Theorem 2.3 (Quantitative Deformation Theorem). Let $X$ be a complete metric space, $f: X \rightarrow \mathbb{R}$ a continuous function, $c \in \mathbb{R}, A$ a closed subset of $X$, and $\delta, \sigma>0$ such that

$$
c-2 \delta \leq f(u) \leq c+2 \delta, \quad d(u, A) \leq \delta / \sigma \quad \Longrightarrow \quad|d f|(u)>2 \sigma .
$$

Then, there exists $\eta: X \times[0,1] \rightarrow X$ continuous with:

(a) $d(\eta(u, t), u) \leq(\delta / \sigma) t$;

(b) $\eta(u, t) \neq u \Longrightarrow f(\eta(u, t))<f(u)$;

(c) $u \in A, c-\delta \leq f(u) \leq c+\delta \Longrightarrow f(\eta(u, t)) \leq f(u)-(f(u)-c+\delta) t$. 
Theorem 2.4 (Quantitative Noncritical Interval Theorem). Let $X$ be $a$ complete metric space, $f: X \rightarrow \mathbb{R}$ be a continuous function, $a, b \in \mathbb{R}$ with $a<b, \delta>0$, and $\sigma>0$ such that

$$
a-\delta \leq f(u) \leq b+\delta \Longrightarrow|d f|(u)>\sigma .
$$

Then, there exists $\eta: X \times[0,1] \rightarrow X$ continuous with:

(a) $d(\eta(u, t), u) \leq \frac{b-a}{\sigma} t$;

(b) $\eta(u, t) \neq u \Longrightarrow f(\eta(u, t))<f(u)$;

(c) $a \leq f(u) \leq b \Longrightarrow f(\eta(u, t)) \leq f(u)-(f(u)-a) t$.

\section{Estimating the weak slope in the presence of linking.}

In this section, $X$ is a metric space endowed with the metric $d$ and $f: X \rightarrow \mathbb{R}$ is a continuous function. If $A, B$ are subsets of $X$, we denote by

$$
d(A, B):=\inf \{d(u, v): u \in A, v \in B\}
$$

the distance between $A$ and $B$, with the convention that $d(A, \emptyset)=+\infty$.

For $c \in \mathbb{R}$, we use the notations

$$
f^{c}:=\{u \in X: f(u)<c\}, \quad f_{c}:=\{u \in X: f(u)>c\},
$$

to denote the open sublevel and upperlevel sets of $f$, respectively.

We say that a (nonempty) subset $B$ of $X$ is contractible in $X$ if there exists a continuous $\psi: B \times[0,1] \rightarrow X$ such that $\psi(u, 0)=u$ for every $u \in B$, and $\psi(u, 1)=u_{0}$ for some $u_{0} \in X$ and every $u \in B$. Such a continuous map $\psi$ will be called a contraction of $B$ in $X$.

We introduce the notion of linking of two subsets of $X$, which is a simplified version of the notion given in [19].

Definition 3.1. Let $X$ be a metric space, and $A, B$ two subsets of $X$. We say that $B$ links $A$ if $B$ is contractible in $X, B \cap A=\emptyset$, and $\psi(B \times[0,1]) \cap A \neq$ $\emptyset$ for every contraction $\psi$ of $B$ in $X$.

In the remainder of this section, we shall consider two subsets $A$ and $B$ of $X$ such that $B$ links $A$. We set:

$$
a:=\inf _{A} f, \quad b_{0}:=\sup _{B} f,
$$

and, denoting by $\Phi_{B}$ the set of contractions of $B$ in $X$ :

$$
b:=\inf _{\psi \in \Phi_{B}} \sup _{B \times[0,1]}(f \circ \psi) .
$$

Of course, $b_{0} \leq b$, and $a \leq b$ since $B$ links $A$.

Theorem 3.2. Assume that $b \in \mathbb{R}, b_{0} \leq a$, and $b-a \leq \delta / 2$ for some $\delta>0$. Then, for any $\sigma>0$ there exists $u \in X$ with

$$
b-2 \delta \leq f(u) \leq b+2 \delta, \quad d(u, A) \leq 2 \delta / \sigma, \quad \text { and } \quad|d f|(u) \leq 2 \sigma .
$$


Proof. We argue by contradiction. Assume that for some $\sigma>0$ we have $u \in X, \quad b-2 \delta \leq f(u) \leq b+2 \delta, \quad d(u, A) \leq 2 \delta / \sigma \quad \Longrightarrow \quad|d f|(u)>2 \sigma$.

Let $\eta: X \times[0,1] \rightarrow X$ continuous be given by Theorem 2.3 (with $A$ replaced by $\{u \in X: d(u, A) \leq \delta / \sigma\})$ such that:

$$
d(\eta(u, t), u) \leq(\delta / \sigma) t, \quad \eta(u, t) \neq u \Longrightarrow f(\eta(u, t))<f(u),
$$

$[d(u, A) \leq \delta / \sigma, b-\delta \leq f(u) \leq b+\delta] \Longrightarrow f(\eta(u, t)) \leq f(u)-(f(u)-b+\delta) t$.

Let $\psi_{0} \in \Phi_{B}$ with $\sup _{B \times[0,1]}\left(f \circ \psi_{0}\right) \leq b+\delta$ and define $\psi \in \Phi_{B}$ by

$$
\psi(u, t)= \begin{cases}u & \text { if } 0 \leq t \leq 3 / 4 \\ \psi_{0}(u, 4 t-3) & \text { if } 3 / 4 \leq t \leq 1,\end{cases}
$$

so that, of course, $\sup _{B \times[0,1]}(f \circ \psi) \leq b+\delta$. Finally, define $\tilde{\psi} \in \Phi_{B}$ by

$$
\tilde{\psi}(u, t)=\eta(\psi(u, t), t) .
$$

Let $(u, t) \in B \times[0,1]$ such that $\tilde{\psi}(u, t) \in A$. Then, $t>3 / 4$ for, if $t \in[0,3 / 4]$ we have $\tilde{\psi}(u, t)=\eta(u, t)$, so that if $\tilde{\psi}(u, t) \neq u$ then $f(\tilde{\psi}(u, t))<f(u) \leq$ $b_{0} \leq a$ and $\tilde{\psi}(u, t) \notin A$. On the other hand, we have $d(\psi(u, t), A) \leq \delta / \sigma$, hence

$$
\begin{aligned}
f(\tilde{\psi}(u, t)) & =f(\eta(\psi(u, t), t)) \\
& \leq f(\psi(u, t))-(f(\psi(u, t))-b+\delta / 2) t<b-\delta / 2 \leq a,
\end{aligned}
$$

contradicting the fact that $\tilde{\psi}(u, t) \in A$.

Theorem 3.3. Let $(X, d)$ be a complete metric space, $f: X \rightarrow \mathbb{R}$ a continuous function, and $A, B$ two subsets of $X$ such that $B$ links $A$. Set:

$$
a:=\inf _{A} f, \quad b_{0}:=\sup _{B} f, \quad b:=\inf _{\psi \in \Phi_{B}} \sup _{B \times[0,1]}(f \circ \psi),
$$

and assume that $a, b \in \mathbb{R}$.

(a) Assume that $b_{0} \leq a$. Then, there exists a sequence $\left(u_{h}\right) \subset X$ with

$$
f\left(u_{h}\right) \rightarrow b \quad \text { and } \quad|d f|\left(u_{h}\right) \rightarrow 0 ;
$$

if, moreover, $b=a$ we can require $\left(u_{h}\right)$ to satisfy also $d\left(u_{h}, A\right) \rightarrow 0$. Consequently, if $f$ satisfies condition $(P S)_{b}$, there exists $u \in X$ such that $f(u)=b,|d f|(u)=0$ (and $u \in \bar{A}$ if $b=a$ ).

(b) Assume that $b_{0}>a$. If either: (i) $0<\alpha \leq d\left(B \cap f_{a}, A\right)$ or: (ii) $0<\alpha \leq d\left(B, f^{b_{0}} \cap A\right)$, and if $\sigma>\left(b_{0}-a\right) / \alpha$, then, for any $\delta>0$ there exists $u \in X$ with

$$
a-\delta \leq f(u) \leq b+\delta \quad \text { and } \quad|d f|(u) \leq \sigma .
$$


Proof. (a) Apply Theorem 3.2 with $\delta=\delta_{h}:=1 / h^{2}, \sigma=\sigma_{h}:=1 / h$, and either the given $A$ if $b=a$, or $A$ replaced by $\tilde{A}:=f_{b-1 / 2 h^{2}}$ if $b>a$. In the latter case, it is indeed clear that $B \cap \tilde{A}=\emptyset$ for large $h$ because $b_{0} \leq a<b$, while $\psi(B \times[0,1]) \cap \tilde{A} \neq \emptyset$ for every $\psi \in \Phi_{B}$, by the definition of $b$. (This shows that (3.4) holds whenever $b \in \mathbb{R}, b>b_{0}$, without any explicit reference to a set $A$ such that $B$ links $A$.)

(b) If $b>b_{0}$, the conclusion is given by (a). Hence, we now assume that $b=b_{0}$. Observe that $b_{0}>a$ means that $B \cap f_{a}$ and $A \cap f^{b_{0}}$ are nonempty, so that $\alpha<+\infty$ - while $\sigma>0$.

Arguing by contradiction, we assume that for some $\delta>0$

$$
u \in X, \quad a-2 \delta \leq f(u) \leq b+2 \delta \quad \Longrightarrow \quad|d f|(u)>\sigma,
$$

and that $\delta$ is so small that $b-a+2 \delta \leq \sigma \alpha$. Let $\eta: X \times[0,1] \rightarrow X$ be given by Theorem 2.4 (with $a$ replaced by $a-\delta$ and $b$ by $b+\delta$ ) such that:

$$
\begin{gathered}
d(\eta(u, t), u) \leq \frac{b-a+2 \delta}{\sigma} t, \quad \eta(u, t) \neq u \Longrightarrow f(\eta(u, t))<f(u), \quad \text { and } \\
a-\delta \leq f(u) \leq b+\delta \Longrightarrow f(\eta(u, t)) \leq f(u)-(f(u)-a+\delta) t .
\end{gathered}
$$

Let $(b-a+\delta) /(b-a+2 \delta)<\varepsilon<1$ be fixed; then,

$$
f(u) \leq b+\delta, \varepsilon \leq t \leq 1 \Longrightarrow f(\eta(u, t))<a .
$$

Indeed, if $a \leq f(u) \leq b+\delta$ and $t \in[\varepsilon, 1]$, we have:

$$
f(\eta(u, t)) \leq(1-t) f(u)+(a-\delta) t \leq b+\delta-(b-a+2 \delta) \varepsilon<a,
$$

while (3.5) clearly holds whenever $f(u)<a$.

Now, let $\psi_{0} \in \Phi_{B}$ with $\sup _{B \times[0,1]}\left(f \circ \psi_{0}\right) \leq b+\delta$, and define $\psi \in \Phi_{B}$ by

$$
\psi(u, t)= \begin{cases}u & \text { if } 0 \leq t \leq \varepsilon \\ \psi_{0}\left(u, \frac{t-\varepsilon}{1-\varepsilon}\right) & \text { if } \varepsilon \leq t \leq 1 ;\end{cases}
$$

finally, define $\tilde{\psi} \in \Phi_{B}$ by

$$
\tilde{\psi}(u, t)=\eta(\psi(u, t), t) .
$$

We show that $\tilde{\psi}(B \times[0,1]) \cap A=\emptyset$, contradicting the fact that $B$ links $A$.

Let $u \in B$. For $t \in[0, \varepsilon]$ we have $\tilde{\psi}(u, t)=\eta(u, t)$, so that

$$
d(\tilde{\psi}(u, t), u)=d(\eta(u, t), u) \leq \frac{b-a+2 \delta}{\sigma} \varepsilon<\alpha,
$$

while

$$
\tilde{\psi}(u, t)=\eta(u, t) \neq u \Longrightarrow f(\tilde{\psi}(u, t))<f(u) .
$$

In case (i), (3.6) shows that $\tilde{\psi}(u, t) \notin A$ whenever $f(u)>a$, while (3.7) shows that $\tilde{\psi}(u, t) \notin A$ whenever $f(u) \leq a$ (recall that $B \cap A=\emptyset$ ). In case 
(ii), (3.6) and (3.7) show that $\tilde{\psi}(u, t) \notin A$ since $f(u) \leq b_{0}$. For $t \in[\varepsilon, 1]$ we have:

$$
f(\tilde{\psi}(u, t))=f\left(\eta\left(\psi_{0}\left(u, \frac{t-\varepsilon}{1-\varepsilon}\right), t\right)\right)<a
$$

according to (3.5), so that again, $\tilde{\psi}(u, t) \notin A$.

Theorem 3.3 (a) is similar to the "usual" min-max principle in the presence of linking, compare with [8, Theorem 3.7]. We now show how Theorem 3.3 can be used to obtain the existence of critical points of $f$, even in the case when (1.1) is not satisfied.

Corollary 3.8. Let $X$ be a complete metric space, $f: X \rightarrow \mathbb{R}$ continuous, and $\left(A_{h}\right),\left(B_{h}\right)$ two sequences of subsets of $X$ such that for each $h$ :

$$
d\left(B_{h}, A_{h}\right)>0 \quad \text { and } \quad B_{h} \text { links } A_{h} .
$$

Set:

$$
a_{h}:=\inf _{A_{h}} f, \quad b_{h 0}:=\sup _{B_{h}} f, \quad b_{h}:=\inf _{\psi \in \Phi_{h}} \sup _{B_{h} \times[0,1]}(f \circ \psi),
$$

where $\Phi_{h}$ denotes the set of contractions of $B_{h}$ in $X$,

$$
d_{h}:=d\left(B_{h} \cap f_{a_{h}}, A_{h}\right), \quad d_{h 0}:=d\left(B_{h}, f^{b_{h 0}} \cap A_{h}\right) .
$$

Assume that $b_{h}, a_{h} \in \mathbb{R}$, eventually, and that either:

$$
\limsup _{h \rightarrow \infty} \frac{b_{h 0}-a_{h}}{d_{h}} \leq 0, \quad \text { or: } \quad \limsup _{h \rightarrow \infty} \frac{b_{h 0}-a_{h}}{d_{h 0}} \leq 0
$$

(with the convention that $\frac{1}{+\infty}=0$ ). Finally, let

$$
b:=\liminf _{h \rightarrow \infty} b_{h}, \quad a:=\limsup _{h \rightarrow \infty} a_{h},
$$

and assume that $a, b \in \mathbb{R}$ and that $f$ satisfies condition $(P S)_{c}$ for all $c \in$ $[a, b]$.

Then, there exists $u \in X$ such that $|d f|(u)=0$ and $f(u) \in[a, b]$.

Proof. Let $h$ be so large that $b_{h}, a_{h} \in \mathbb{R}$. Of course, $d_{h}=+\infty$ (resp., $\left.d_{h 0}=+\infty\right)$ if and only if $b_{h 0} \leq a_{h}$. In this case, apply Theorem 3.3 (a) to obtain $u_{h} \in X$ with $\left|f\left(u_{h}\right)-b_{h}\right| \leq 1 / h$ and $|d f|\left(u_{h}\right) \leq 1 / h$.

Whenever $d_{h}<+\infty$ (resp., $d_{h 0}<+\infty$ ), observe first that $d_{h}>0$ (resp., $\left.d_{h 0}>0\right)$ since $d\left(B_{h}, A_{h}\right)>0$. Let $\varepsilon_{h}>0$ with $\varepsilon_{h} \rightarrow 0$ be such that

$$
\frac{b_{h 0}-a_{h}}{d_{h}} \leq \varepsilon_{h} \quad\left(\text { resp. }, \frac{b_{h 0}-a_{h}}{d_{h 0}} \leq \varepsilon_{h}\right),
$$

and apply Theorem 3.3 (b) with $\alpha=\alpha_{h}:=d_{h}$ (resp., $\alpha=\alpha_{h}:=d_{h 0}$ ) and $\sigma=\sigma_{h}:=\varepsilon_{h}+1 / h$ to obtain $u_{h} \in X$ with

$$
a_{h}-1 / h \leq f\left(u_{h}\right) \leq b_{h}+1 / h \quad \text { and } \quad|d f|\left(u_{h}\right) \leq \sigma_{h} .
$$


The conclusion follows, using the Palais-Smale condition (see Definition 2.2).

Remark 3.9. The results of this section are nonsmooth variants of results of $[\mathbf{1 7}]$.

\section{Changing the metric.}

In this section, $X$ is a metric space endowed with the metric $d$, and $f: X \rightarrow$ $\mathbb{R}$ is a continuous function. We describe a general procedure which allows to deduce new results from those of the preceding section, by a change of the metric of $X$.

Theorem 4.1. Let $(X, d)$ be a metric space, $\tilde{A}$ be a nonempty subset of $X$, and $\beta:[0,+\infty[\rightarrow] 0,+\infty[$ be continuous. Then, there exists a metric $\tilde{d}$ on $X$ which is topologically equivalent to $d$ and such that:

(a) For any subset $B$ of $X$, it holds

$$
\tilde{d}(B, \tilde{A}) \geq \int_{0}^{d(B, \tilde{A})} \beta(t) d t .
$$

Consequently, if $\int_{0}^{+\infty} \beta(t) d t=+\infty$, then $(X, \tilde{d})$ is complete if (and only if $)(X, d)$ is complete;

(b) if $f: X \rightarrow \mathbb{R}$ is a continuous function, and we denote by $|\tilde{d} f|$ the weak slope of $f$ with respect to the metric $\tilde{d}$, it holds

$$
|\tilde{d} f|(u)=\frac{|d f|(u)}{\beta(d(u, \tilde{A}))} \quad \text { for every } u \in X .
$$

Proof. Let $Y$ be a Banach space such that $X$ is isometrically embedded in $Y$, according to the Arens-Eells Theorem (see, e.g., [11, in 2.5.16, p. 110]). For $u, v \in Y$, we denote by $\Gamma_{u, v}$ the set of $C^{1}$-paths $\gamma:[0,1] \rightarrow Y$ with $\gamma(0)=u, \gamma(1)=v$, and we set

$$
\tilde{d}(u, v):=\inf _{\gamma \in \Gamma u, v} \int_{0}^{1} \beta(d(\gamma(t), \tilde{A}))\left\|\gamma^{\prime}(t)\right\| d t,
$$

where $d$ also stands for the distance associated to the norm. It is easy to verify that $\tilde{d}$ is a metric on $Y$. Considering line segments, we see that if $B \subset Y$ is norm-bounded there exists $\delta>0$ such that

$$
\tilde{d}(u, v) \leq \delta\|u-v\| \quad \text { for every } u, v \in B .
$$

Also, if $u \in Y$ and $r>0$ are fixed there exists $\hat{\delta}>0$ such that

$$
\|u-v\| \geq r \Longrightarrow \tilde{d}(u, v) \geq \hat{\delta} .
$$

It follows that the restriction of $\tilde{d}$ to $X$ is topologically equivalent to $d$, and that $(X, d)$ is complete whenever $(X, \tilde{d})$ is. 
To prove (a), we may assume that $B \neq \emptyset$ (otherwise, (4.2) is trivially satisfied). Let $u \in \tilde{A}, v \in B$, and $\gamma \in \Gamma_{u, v}$. For $t \in[0,1]$ set $\rho(t):=d(\gamma(t), \tilde{A})$, so that $\rho(0)=0, \rho(1)=d(v, \tilde{A})$. Being locally Lipschitz continuous on $[0,1], \rho$ is almost everywhere differentiable, and $\left|\rho^{\prime}(t)\right| \leq\left\|\gamma^{\prime}(t)\right\|$ for almost every $t \in] 0,1[$. We thus have, through a change of variable (see, e.g., $[\mathbf{1 1}$, Theorem 3.2.5]):

$$
\int_{0}^{1} \beta(d(\gamma(t), \tilde{A}))\left\|\gamma^{\prime}(t)\right\| d t \geq \int_{0}^{1} \beta(\rho(t))\left|\rho^{\prime}(t)\right| d t \geq \int_{0}^{d(v, \tilde{A})} \beta(t) d t .
$$

From the definition of $\tilde{d}$ and since $\gamma$ is arbitrary in $\Gamma_{u, v}$, we obtain that

$$
\tilde{d}(u, v) \geq \int_{0}^{d(v, \tilde{A})} \beta(t) d t \geq \int_{0}^{d(B, \tilde{A})} \beta(t) d t .
$$

Since $u$ and $v$ are arbitrary in $\tilde{A}$ and $B$, respectively, (4.2) follows.

We now observe that for every $R>0$ there exists $\tilde{\delta}>0$ such that

$$
d\left(u_{i}, \tilde{A}\right) \leq R, i=1,2 \Longrightarrow \tilde{d}\left(u_{1}, u_{2}\right) \geq \tilde{\delta} \min \left\{1,\left\|u_{1}-u_{2}\right\|\right\} .
$$

Indeed, for $\gamma \in \Gamma_{u_{1}, u_{2}}$ let $t_{\gamma}:=\sup \{t \in[0,1]: d(\gamma(s), \tilde{A}) \leq R+1$ for all $s \in$ $[0, t]\} \in] 0,1]$, and let $\tilde{\delta}:=\min _{[0, R+1]} \beta$. Then,

$$
\int_{0}^{t_{\gamma}} \beta(d(\gamma(t), \tilde{A}))\left\|\gamma^{\prime}(t)\right\| d t \geq \tilde{\delta}\left\|u_{1}-\gamma\left(t_{\gamma}\right)\right\| \geq \tilde{\delta} \min \left\{1,\left\|u_{1}-u_{2}\right\|\right\},
$$

considering the cases $t_{\gamma}<1, t_{\gamma}=1$, and (4.3) follows since $\gamma$ is arbitrary in $\Gamma_{u_{1}, u_{2}}$.

Thus, if $\int_{0}^{+\infty} \beta(t) d t=+\infty$ and $\left(u_{h}\right)$ is a Cauchy sequence in $(X, \tilde{d}),(4.2)$ shows that $\left(d\left(u_{h}, \tilde{A}\right)\right)$ is bounded, and (4.3) shows that $\left(u_{h}\right)$ is a Cauchy sequence in $(X, d)$. Hence, $(X, \tilde{d})$ is complete if $(X, d)$ is complete.

Assertion (b) is derived from the definitions of $\tilde{d}$ and $|\tilde{d} f|$ (see Definition 2.1); we give the proof for the reader's convenience. In a standard way, we need to establish both inequalities. We still consider $X$ as embedded in the Banach space $Y$.

Fix $u \in X, \varepsilon>0$, and assume that $|d f|(u)>\sigma>0$. Let $\delta>0$ and $\mathcal{H}: B(u ; \delta) \times[0, \delta] \rightarrow X$ such that

$$
\|\mathcal{H}(v, t)-v\| \leq t, \quad f(\mathcal{H}(v, t)) \leq f(v)-\sigma t ;
$$

we may assume that $\delta$ is so small that

$$
\beta(d(v, \tilde{A})) \leq \beta(d(u, \tilde{A}))+\varepsilon \quad \text { for all } v \in B(u ; 2 \delta) .
$$

Let $0<\tilde{\delta} \leq \delta(\beta(d(u, \tilde{A}))+\varepsilon$ ) such that $\tilde{B}(u ; \tilde{\delta}) \subset B(u ; \delta)$ (where $\tilde{B}$ stands for the ball with respect to the metric $\tilde{d})$, and define $\tilde{\mathcal{H}}: \tilde{B}(u ; \tilde{\delta}) \times[0, \tilde{\delta}] \rightarrow X$ 
by

$$
\tilde{\mathcal{H}}(v, t)=\mathcal{H}\left(v, \frac{t}{\beta(d(u, \tilde{A}))+\varepsilon}\right) .
$$

For $(v, t) \in \tilde{B}(u ; \tilde{\delta}) \times[0, \tilde{\delta}]$ and $s \in[0,1]$, let $\gamma(s):=v+s(\tilde{\mathcal{H}}(v, t)-v) \in$ $B(u ; 2 \delta)$. Then,

$$
\tilde{d}(\tilde{\mathcal{H}}(v, t), v) \leq \int_{0}^{1} \beta(d(\gamma(s), \tilde{A}))\left\|\gamma^{\prime}(s)\right\| d s \leq t
$$

and

$$
f(\tilde{\mathcal{H}}(v, t)) \leq f(v)-\frac{\sigma}{\beta(d(u, \tilde{A}))+\varepsilon} t .
$$

It follows that $|\tilde{d} f|(u) \geq \frac{\sigma}{\beta(d(u, \tilde{A}))+\varepsilon}$, hence $|\tilde{d} f|(u) \geq \frac{\sigma}{\beta(d(u, \tilde{A}))}$ since $\varepsilon>0$ is arbitrary. Consequently, $|\tilde{d} f|(u) \geq \frac{|d f|(u)}{\beta(d(u, \tilde{A}))}$, the inequality being obvious whenever $|d f|(u)=0$.

Conversely, let $u \in X$ and $0<\varepsilon<\beta(d(u, \tilde{A}))$ be fixed, and let $\delta_{0}>0$ such that

$$
\beta(d(v, \tilde{A})) \geq \beta(d(u, \tilde{A}))-\varepsilon \quad \text { for all } v \in B\left(u ; 3 \delta_{0}\right) .
$$

Assume that $|\tilde{d} f|(u)>\sigma>0$. Let $\tilde{\delta}>0$ and $\tilde{\mathcal{H}}: \tilde{B}(u ; \tilde{\delta}) \times[0, \tilde{\delta}] \rightarrow X$ such that

$$
\tilde{d}(\tilde{\mathcal{H}}(v, t), v) \leq t, \quad f(\tilde{\mathcal{H}}(v, t)) \leq f(v)-\sigma t ;
$$

we may assume that $\tilde{\delta}$ is so small that $\tilde{B}(u ; 2 \tilde{\delta}) \subset B\left(u ; \delta_{0}\right)$. For $(v, t) \in$ $\tilde{B}(u ; \tilde{\delta}) \times[0, \tilde{\delta}]$ and $\gamma \in \Gamma_{v, \widetilde{\mathcal{H}}(v, t)}$, let $\tilde{s}:=\sup \left\{s \in[0,1]: \gamma(r) \in B\left(u ; 3 \delta_{0}\right)\right.$ for all $0 \leq r \leq s\} \in] 0,1]$. We have:

$$
\begin{aligned}
\int_{0}^{\tilde{s}} \beta(d(\gamma(s), \tilde{A}))\left\|\gamma^{\prime}(s)\right\| d s & \geq(\beta(d(u, \tilde{A}))-\varepsilon)\|\gamma(\tilde{s})-v\| \\
& \geq(\beta(d(u, \tilde{A}))-\varepsilon)\|\tilde{\mathcal{H}}(v, t)-v\| .
\end{aligned}
$$

We deduce that

$$
(\beta(d(u, \tilde{A}))-\varepsilon)\|\tilde{\mathcal{H}}(v, t)-v\| \leq \tilde{d}(\tilde{\mathcal{H}}(v, t), v) \leq t .
$$

Let now $\delta>0$ such that $(\beta(d(u, \tilde{A}))-\varepsilon) \delta \leq \tilde{\delta}$ and $B(u ; \delta) \subset \tilde{B}(u ; \tilde{\delta})$, and define $\mathcal{H}: B(u ; \delta) \times[0, \delta] \rightarrow X$ by

$$
\mathcal{H}(v, t)=\tilde{\mathcal{H}}(v,(\beta(d(u, \tilde{A}))-\varepsilon) t)
$$

Then,

$$
\|\mathcal{H}(v, t)-v\| \leq t \quad \text { and } \quad f(\mathcal{H}(v, t)) \leq f(v)-\sigma(\beta(d(u, \tilde{A}))-\varepsilon) t .
$$


It follows that $|\tilde{d} f|(u) \geq \sigma(\beta(d(u, \tilde{A}))-\varepsilon)$, hence $|\tilde{d} f|(u) \geq \sigma \beta(d(u, \tilde{A}))$ since $\varepsilon>0$ is arbitrary. Consequently, $|d f|(u) \geq \beta(d(u, \tilde{A}))|\tilde{d} f|(u)$, the inequality being obvious whenever $|\tilde{d} f|(u)=0$.

Remark 4.4. In the case when $X$ is a Banach space, $\tilde{A}$ is the origin, and $\beta(t)=1 /(1+t)$, the metric $\tilde{d}$ defined in the above proof is the Cerami metric, see [5], [10, p. 138], where it is used in the context of critical point theory for "smooth" functions. This metric is used in [1] in the context of the critical point theory for continuous functions defined on complete metric spaces developed in $[\mathbf{9}, \mathbf{8}]$. Since $(X, \tilde{d})$ is complete (see Theorem 4.1 (a)), and the Palais-Smale condition "with weight" $\beta(\|u\|)^{-1}=1+\|u\|$ (see, e.g., [16] for the terminology) is just the condition of Definition 2.2 in $(X, \tilde{d})$, existence results of critical points of a continuous function $f: X \rightarrow \mathbb{R}$ readily follow from the general theory (see Theorem 3.3 (a)).

Here, we show how the main abstract results of $[\mathbf{1 8}]$ can be obtained from the results of Section 3, just changing metric according to the previous theorem.

Theorem 4.5. Let $X$ be a complete metric space, $f: X \rightarrow \mathbb{R}$ continuous, and $A, B$ two subsets of $X$ such that $B$ links $A$. Set:

$$
a:=\inf _{A} f, \quad b_{0}:=\sup _{B} f, \quad b:=\inf _{\psi \in \Phi_{B}} \sup _{B \times[0,1]}(f \circ \psi),
$$

where $\Phi_{B}$ is the set of contractions of $B$ in $X$, and assume that $a, b \in \mathbb{R}$ and $b_{0}>a$. If $0<\rho<d\left(B \cap f_{a}, A\right)$ (resp., $0<\rho<d\left(B, A \cap f^{b_{0}}\right)$ ) and $\beta:[0,+\infty[\rightarrow] 0,+\infty[$ is a continuous function such that

$$
\int_{0}^{+\infty} \beta(t) d t=+\infty \quad \text { and } \quad \int_{0}^{\rho} \beta(t) d t \geq b_{0}-a,
$$

then, for any $\delta>0$ there exists $u \in X$ with

$a-\delta \leq f(u) \leq b+\delta$ and $|d f|(u) \leq \beta(d(u, A))$ (resp., $|d f|(u) \leq \beta(d(u, B)))$.

Proof. We consider the case $0<\rho<d\left(B \cap f_{a}, A\right)$. Let $\tilde{d}$ be the metric given by Theorem 4.1 , with $\tilde{A}:=A$. Then, $(X, \tilde{d})$ is complete and

$$
\tilde{d}\left(B \cap f_{a}, A\right) \geq \int_{0}^{d\left(B \cap f_{a}, A\right)} \beta(t) d t>\int_{0}^{\rho} \beta(t) d t \geq b_{0}-a>0,
$$

while $|\tilde{d} f|(u)=\frac{|d f|(u)}{\beta(d(u, A))}$ for every $u \in X$. Applying Theorem 3.3 (b) in $(X, \tilde{d})$ with $\alpha:=\tilde{d}\left(B \cap f_{a}, A\right)$ and $\sigma:=1$ yields the conclusion.

In the case $0<\rho<d\left(B, A \cap f^{b_{0}}\right)$, consider the metric $\tilde{d}$ associated with $\beta$ and $\tilde{A}:=B$.

Remark 4.6. Letting $\beta(t) \equiv \sigma$, a positive constant, we recover Theorem 3.3 (b). 
Corollary 4.7. Let $X$ be a complete metric space, $f: X \rightarrow \mathbb{R}$ continuous, and $\left(A_{h}\right),\left(B_{h}\right)$ two sequences of subsets of $X$ such that for each $h$ :

$$
d\left(B_{h}, A_{h}\right)>0 \quad \text { and } \quad B_{h} \text { links } A_{h} .
$$

Set:

$$
a_{h}:=\inf _{A_{h}} f, \quad b_{h 0}:=\sup _{B_{h}} f, \quad b_{h}:=\inf _{\psi \in \Phi_{h}} \sup _{B_{h} \times[0,1]}(f \circ \psi),
$$

where $\Phi_{h}$ denotes the set of contractions of $B_{h}$ in $X$,

$$
d_{h}:=d\left(B_{h} \cap f_{a_{h}}, A_{h}\right), \quad d_{h 0}:=d\left(B_{h}, f^{b_{h 0}} \cap A_{h}\right) .
$$

Assume that $b_{h}, a_{h} \in \mathbb{R}$, eventually, and that for some $\mu \geq 0$ :

$$
\limsup _{h \rightarrow \infty} \frac{b_{h 0}-a_{h}}{d_{h}^{\mu+1}} \leq 0, \quad\left(\text { resp., } \limsup _{h \rightarrow \infty} \frac{b_{h 0}-a_{h}}{d_{h 0}^{\mu+1}} \leq 0\right)
$$

(with the convention that $\frac{1}{+\infty}=0$ ).

Then, there is a sequence $\left(u_{h}\right)$ in $X$ such that

$$
a_{h}-1 / h \leq f\left(u_{h}\right) \leq b_{h}+1 / h
$$

and

$$
\frac{|d f|\left(u_{h}\right)}{\left(d\left(u_{h}, A_{h}\right)+1\right)^{\mu}} \rightarrow 0, \quad\left(\text { resp. }, \frac{|d f|\left(u_{h}\right)}{\left(d\left(u_{h}, B_{h}\right)+1\right)^{\mu}} \rightarrow 0\right) .
$$

Proof. As in the proof of Corollary 3.8, we let $h$ be so large that $b_{h}, a_{h} \in \mathbb{R}$ and we observe that $d_{h}=+\infty$ (resp., $d_{h 0}=+\infty$ ) if and only if $b_{h 0} \leq a_{h}$. In this case, apply Theorem 3.3 (a) to obtain $u_{h} \in X$ with $\left|f\left(u_{h}\right)-b_{h}\right| \leq 1 / h$ and

$|d f|\left(u_{h}\right) \leq 1 / h \leq\left(d\left(u_{h}, A_{h}\right)+1\right)^{\mu} / h \quad$ (resp., $\left.|d f|\left(u_{h}\right) \leq\left(d\left(u_{h}, B_{h}\right)+1\right)^{\mu} / h\right)$.

If $d_{h}<+\infty$ (resp., $d_{h 0}<+\infty$ ), then $d_{h}>0$ (resp., $d_{h 0}>0$ ) because $d\left(B_{h}, A_{h}\right)>0$. Define (similarly as in the proof of [18, Corollary 2.3]) a continuous nondecreasing function $\beta_{h}:[0,+\infty[\rightarrow] 0,+\infty[$ by

$$
\beta_{h}(t)=(\mu+1)\left(b_{h 0}-a_{h}\right)(t+1)^{\mu} / D_{h}^{\mu+1},
$$

where $D_{h}:=d_{h} / 2$ (resp., $\left.D_{h}:=d_{h 0} / 2\right)$. We have:

$$
\int_{0}^{D_{h}} \beta_{h}(t) d t=\left(b_{h 0}-a_{h}\right) \frac{\left(D_{h}+1\right)^{\mu+1}-1}{D_{h}^{\mu+1}} \geq b_{h 0}-a_{h}>0 .
$$

Applying Theorem 4.5, we obtain $u_{h} \in X$ with $a_{h}-1 / h \leq f\left(u_{h}\right) \leq b_{h}+1 / h$ and

$$
\left.|d f|\left(u_{h}\right) \leq \beta_{h}\left(d\left(u_{h}, A_{h}\right)\right) \quad \text { (resp., } \quad|d f|\left(u_{h}\right) \leq \beta_{h}\left(d\left(u_{h}, B_{h}\right)\right)\right) .
$$

Dividing by $\left(d\left(u_{h}, A_{h}\right)+1\right)^{\mu}$ (resp., $\left.\left(d\left(u_{h}, B_{h}\right)+1\right)^{\mu}\right)$, and taking the lim sup as $h \rightarrow \infty$ yields the conclusion. 
Remark 4.8. Theorem 4.5 extends Schechter's [18, Theorems 2.1, 2.2], where $X$ is a Banach space, $f$ is of class $C^{1}$, and $\beta$ is a positive nondecreasing function (observe that if $\inf \beta>0$, Theorem 4.5 holds without assuming $b_{0}>a$, thanks to Theorem $3.3(\mathrm{a})$ ). Corollary 4.7 extends [18, Corollary $2.3]$.

\section{Some corollaries in Banach spaces.}

In this section, $X$ is a Banach space and $f: X \rightarrow \mathbb{R}$ is continuous. We give some corollaries of the results of Sections 3 and 4 , which are useful in applications. First, we give standard examples of linking subsets, corresponding to the geometrical situations encountered in Rabinowitz' Saddle Point and Generalized Mountain Pass Theorems, see [15].

Example 5.1. Let $X$ be a Banach space which splits into the direct sum $X=Y \oplus Z$, with $Y$ finite dimensional. Set

$$
A:=Z ; \quad B_{R}:=\partial B(0 ; R) \cap Y, \quad R>0 .
$$

Then, $B_{R}$ links $A$. Moreover, if $f: X \rightarrow \mathbb{R}$ and we set:

$$
b:=\inf _{\psi \in \Phi_{B_{R}}} \sup _{B_{R} \times[0,1]}(f \circ \psi), \quad \tilde{b}:=\inf _{\varphi \in \Gamma} \sup _{\bar{B}(0 ; R) \cap Y}(f \circ \varphi),
$$

where $\Phi_{B_{R}}$ is the set of contractions of $B_{R}$ in $X$ and

$\Gamma:=\left\{\varphi: \bar{B}(0 ; R) \cap Y \rightarrow X: \varphi\right.$ is continuous and $\varphi(u)=u$ for $\left.u \in B_{R}\right\}$, then $b=\tilde{b}$.

Proof. The fact that $B_{R}$ links $A$ follows from a standard application of Brouwer's topological degree, similar to the proof that $\varphi(\bar{B}(0 ; R) \cap Y) \cap A \neq \emptyset$ for any $\varphi \in \Gamma$, see, e.g., [15, Chapter 5]. Now, if $\psi \in \Phi_{B_{R}}$ with $\psi(u, 1)=u_{0}$, we can define $\varphi \in \Gamma$ by

$$
\varphi(u)= \begin{cases}\psi(R u /\|u\|, 1-\|u\| / R) & \text { if } u \neq 0 \\ u_{0} & \text { if } u=0,\end{cases}
$$

so that $\varphi(\bar{B}(0 ; R) \cap Y)=\psi\left(B_{R} \times[0,1]\right)$ and $\tilde{b} \leq b$. Conversely, if $\varphi \in \Gamma$ define $\psi \in \Phi_{B_{R}}$ by $\psi(u, t)=\varphi((1-t) u)$, so that $\psi\left(B_{R} \times[0,1]\right)=\varphi(\bar{B}(0 ; R) \cap Y)$, and $b \leq \tilde{b}$.

In a similar way, we have:

Example 5.2. Let $X$ be a Banach space, $X=Y \oplus Z$ with $Y$ finite dimensional, and $v_{0} \in Z, v_{0} \neq 0$. For $\rho>0, R>0$, set:

$$
\begin{gathered}
A_{\rho}:=\partial B(0 ; \rho) \cap Z, \\
B_{R}^{\prime}:=\left\{s v_{0}+u: s \geq 0, u \in Y,\left\|s v_{0}+u\right\|=R\right\}, \\
B_{R}:=B_{R}^{\prime} \cup(B(0 ; R) \cap Y) \subset Y \oplus \mathbb{R} v_{0} .
\end{gathered}
$$


Then, $B_{R}$ links $A_{\rho}$ if $R>\rho$.

Theorem 5.3. Let $X$ be a Banach space which splits into the direct sum $X=Y \oplus Z$, with $Y$ finite dimensional, and let $f: X \rightarrow \mathbb{R}$ be a continuous function. Set:

$$
\begin{aligned}
& m_{Y}:=\sup _{u \in Y} \inf _{v \in Z} f(u+v), \\
& m_{Z}:=\inf _{v \in Z} \sup _{u \in Y} f(u+v) .
\end{aligned}
$$

Assume that $m_{Y}, m_{Z} \in \mathbb{R}$, and that $f$ satisfies condition $(P S)_{c}$ for every $c \in\left[m_{Y}, m_{Z}\right]$.

Then, there exists $u \in X$ with $|d f|(u)=0$ and $f(u) \in\left[m_{Y}, m_{Z}\right]$.

Proof. Based on Corollary 3.8, the proof is formally the same as in [17, Theorem 2.11]. For $h \in \mathbb{N}$, let $u_{h} \in Y$ and $v_{h} \in Z$ such that

$$
\inf _{v \in Z} f\left(u_{h}+v\right) \geq m_{Y}-1 / h, \quad \sup _{u \in Y} f\left(u+v_{h}\right) \leq m_{Z}+1 / h,
$$

and set:

$$
A_{h}:=u_{h} \oplus Z, \quad B_{h}:=\left\{u+v_{h}: u \in Y,\left\|u-u_{h}\right\|=h\right\} \subset Y \oplus v_{h} .
$$

Then, $B_{h}$ links $A_{h}$ for every $h$ : See Example 5.1.

Now, set:

$$
\begin{gathered}
a_{h}:=\inf _{A_{h}} f, \quad b_{h}:=\inf _{\psi \in \Phi_{B_{h}} B_{h} \times[0,1]} \sup _{h \rightarrow \psi),}\left(f \circ{ }=\limsup _{h \rightarrow \infty} a_{h}, \quad b:=\liminf _{h \rightarrow \infty} b_{h} .\right.
\end{gathered}
$$

It is immediately seen that $m_{Y} \leq a \leq b \leq m_{Z}$. Since $d\left(B_{h}, A_{h}\right)=h$, the conclusion follows from Corollary 3.8, and the Palais-Smale condition.

Theorem 5.4. Let $X$ be a Banach space which splits into the direct sum $X=Y \oplus Z$, with $Y$ finite dimensional, and let $f: X \rightarrow \mathbb{R}$ be a continuous function. Assume that there exist $\rho>0$ and $m_{0} \in \mathbb{R}$ such that

$$
f(u) \leq m_{0} \leq f(v) \quad \text { for all } u \in Y \text { and all } v \in \partial B(0 ; \rho) \cap Z,
$$

and that there is some $v_{0} \in Z, v_{0} \neq 0$, such that for $R>0$ there exists $m_{R} \in \mathbb{R}$ with

$$
f\left(s v_{0}+u\right) \leq m_{R} \quad \text { for all } s \geq 0, u \in Y,\left\|s v_{0}+u\right\|=R .
$$

(a) Assume that $m:=\sup _{R>0} m_{R} \in \mathbb{R}$ and that $f$ satisfies condition $(P S)_{c}$ for every $c \in\left[m_{0}, m\right]$. Then, there exists $u \in X$ with $|d f|(u)=0$ and $f(u) \in\left[m_{0}, m\right]$.

(b) Assume that for some $\mu \geq 0, m_{R} / R^{\mu+1} \rightarrow 0$ as $R \rightarrow \infty$. Then, there is a sequence $\left(u_{h}\right) \subset X$ such that

$$
\liminf _{h} f\left(u_{h}\right) \geq m_{0} \quad \text { and } \quad \frac{|d f|\left(u_{h}\right)}{\left(\left\|u_{h}\right\|+1\right)^{\mu}} \rightarrow 0 .
$$


Proof. Let $A_{\rho}, B_{R}^{\prime}$, and $B_{R}, R>0$, be defined as in Example 5.2, so that $B_{R}$ links $A_{\rho}$ as soon as $R>\rho$. Let

$$
\begin{gathered}
a_{\rho}:=\inf _{A_{\rho}} f \geq m_{0}, \quad b_{R 0}:=\sup _{B_{R}} f \leq \max \left\{m_{0}, m_{R}\right\}, \\
b_{R}:=\inf _{\psi \in \Phi_{B_{R}} \sup _{B_{R} \times[0,1]}(f \circ \psi) .},
\end{gathered}
$$

We have $B_{R} \cap f_{a_{\rho}} \subset B_{R}^{\prime}$ and $d\left(B_{R}^{\prime}, A_{\rho}\right)=R-\rho$.

(a) Observe that $m \geq m_{0}$ since $B_{\rho}^{\prime} \cap A_{\rho} \neq \emptyset$, and that $b_{R} \leq m$ for all $R>0$ (consider $\psi \in \Phi_{B_{R}}$ with $\left.\psi\left(B_{R} \times[0,1]\right) \subset \overline{\cup_{0<r \leq R} B_{r}^{\prime}}\right)$. Since

$$
\frac{b_{R 0}-a_{\rho}}{d\left(B_{R} \cap f_{a_{\rho}}, A_{\rho}\right)} \leq \frac{m-m_{0}}{R-\rho} \rightarrow 0 \quad \text { as } R \rightarrow \infty,
$$

the conclusion follows from Corollary 3.8 and the Palais-Smale condition.

(b) We have:

$$
\frac{b_{R 0}-a_{\rho}}{d\left(B_{R} \cap f_{a_{\rho}}, A_{\rho}\right)^{\mu+1}} \leq \frac{m_{R}-m_{0}}{(R-\rho)^{\mu+1}} \rightarrow 0 \quad \text { as } R \rightarrow \infty,
$$

and, for every $u \in X$,

$$
\frac{|d f|(u)}{(\|u\|+1)^{\mu}} \leq(\rho+1)^{\mu} \frac{|d f|(u)}{\left(d\left(u, A_{\rho}\right)+1\right)^{\mu}} ;
$$

so, the conclusion follows from Corollary 4.7.

Remark 5.5. Theorems 5.3 and 5.4 are variants of results of Silva [20] and Schechter $[\mathbf{1 7}, \mathbf{1 8}]$ (see also the references in $[\mathbf{1 8}]$ ), dealing with a $C^{1}$ functional $f$. We observe that in the corresponding results of $[\mathbf{1 7}, \mathbf{1 8}]$, it is assumed that either $Y$ or $Z$ is finite dimensional. For the $C^{1}$ version of Theorem 5.3 this is possible, of course, because the statement is "symmetric" with respect to the splitting of the space, and $f$ and $-f$ have the same critical points - which is no more the case, in general, if $f$ is only continuous. As for the $C^{1}$ version of Theorem 5.4, the notion of linking introduced in [19] ensures that $B_{R}$ links $A_{\rho}(R>\rho)$ also when $Z$, rather than $Y$, is finite dimensional; basically, this is due to the fact that the notion of [19] involves contractions $\psi \in \Phi_{X}$ such that $\psi(\cdot, t)$ is a homeomorphism onto $X$ for each $t \in[0,1[$. As mentioned in the introduction, we cannot, in our setting, restrict the class of admissible deformations in this direction.

Remark 5.6. Theorem 5.4 (b) does not assert existence of a critical point of the function $f$. In applications, one has to show that the kind of "PalaisSmale sequence" obtained has a subsequence converging to such a critical point.

Acknowledgment. I thank Marco Degiovanni for a helpful discussion about Section 4. 


\section{References}

[1] G. Arioli and F. Gazzola, Weak solutions of quasilinear elliptic PDE's at resonance, Ann. Fac. Sci. Toulouse, Ser. 6, VI (1997), 573-589.

[2] H. Brezis and L. Nirenberg, Remarks on finding critical points, Comm. Pure Appl. Math., 44 (1991), 939-963.

[3] A. Canino, Multiplicity of solutions for quasilinear elliptic equations, Topol. Methods Nonlinear Anal., 6 (1995), 357-370.

[4] A. Canino and M. Degiovanni, Nonsmooth critical point theory and quasilinear elliptic equations, Proc. NATO ASI \& Séminaire de Mathématiques Supérieures, "Topological Methods in Differential Equations and Inclusions", Montréal 1994, Kluwer, Dordrecht, 1995, 1-50.

[5] G. Cerami, Un criterio di esistenza per $i$ punti critici su varietà illimitate, Rend. Accad. Sc. Lett. Istit. Lombardo, 112 (1978), 332-336.

[6] J.-N. Corvellec, Morse theory for continuous functionals, J. Math. Anal. Appl., 195 (1995), 1050-1072.

[7] J.-N. Corvellec and M. Degiovanni, Nontrivial solutions of quasilinear equations via nonsmooth Morse theory, J. Diff. Equations, 136 (1997), 268-293.

[8] J.-N. Corvellec, M. Degiovanni and M. Marzocchi, Deformation properties for continuous functionals and critical point theory, Topol. Methods Nonlinear Anal., 1 (1993), 151-171.

[9] M. Degiovanni and M. Marzocchi, A critical point theory for nonsmooth functionals, Ann. Mat. Pura Appl., 167 (1994), 73-100.

[10] I. Ekeland, Convexity methods in Hamiltonian mechanics, Springer-Verlag, 1990.

[11] H. Federer, Geometric measure theory, Springer-Verlag, 1969.

[12] A. Ioffe and E. Schwartzman, Metric critical point theory 1. Morse regularity and homotopic stability of a minimum, J. Math. Pures Appl., 75 (1996), 125-153.

[13] _ Metric critical point theory 2. Deformation techniques, preprint, 1996.

[14] G. Katriel, Mountain pass theorems and global homeomorphism theorems, Ann. Inst. H. Poincaré Anal. Non Linéaire, 11 (1994), 189-209.

[15] P.H. Rabinowitz, Minimax methods in critical point theory with applications to differential equations, CBMS Reg. Conf. Series Math., Vol. 65, Amer. Math. Soc., Providence, R.I., 1986.

[16] M. Ramos, Teoremas de enlace na teoria dos pontos críticos, Textos de Matemática, Faculdade de Ciências da Universidade de Lisboa, 1993.

[17] M. Schechter, The intrinsic mountain pass, Pacific J. Math., 171 (1995), 529-544.

[18] _ Critical points when there is no saddle point geometry, Topol. Methods Nonlinear Anal., 6 (1995), 295-308.

[19] M. Schechter and K. Tintarev, Pairs of critical points produced by linking subsets with applications to semilinear elliptic problems, Bull. Soc. Math. Belg., 44 (1992), 249-261.

[20] E.A. de B.e Silva, Linking theorems and applications to semilinear elliptic problems at resonance, Nonlinear Anal., 16 (1991), 455-477. 
[21] M. Willem, Lectures on critical point theory, Trabalho de Mat., Vol. 199, Fundação Univ. Brasilia, Brasilia, 1983.

Received September 12, 1997 and revised March 5, 1998.

Université de Perpignan

66860 Perpignan Cedex

FRANCE

E-mail address: corvellec@univ-perp.fr 